\title{
COVID-19-associated atypical hemolytic uremic syndrome and use of Eculizumab therapy
}

\author{
Jasmeet Gill ${ }^{1}$. Christopher Alan Hebert ${ }^{2}$. Gates Burton Colbert ${ }^{2}$
}

Received: 12 May 2021 / Accepted: 12 July 2021 / Published online: 24 August 2021

(c) Italian Society of Nephrology 2021

\begin{abstract}
There is a high incidence of acute kidney injury with COVID-19 infections. The etiologies of acute kidney injury could be ischemic acute tubular necrosis or a complex process of complement activation leading to thrombotic microangiopathy. We present a case of 32-year-old Hispanic male with a history of heart transplant, admitted with COVID-19 and atypical hemolytic uremic syndrome, which was successfully treated with Eculizumab.
\end{abstract}

Keywords Thrombotic microangiopathy $\cdot$ COVID-19 $\cdot$ Eculizumab $\cdot$ Acute kidney injury

\section{Introduction}

In December 2019, COVID-19 infection caused by severe acute respiratory syndrome-Coronavirus-2 (SARS-CoV-2) initially presented with involvement of the respiratory tract alone. However, over time it has manifested as a complex, multiorgan disorder involving the heart, liver, kidney, lung and brain. The pathophysiology is rooted in the over-activation of the immune system with cytokine storm and activated complement system leading to multiorgan failure [1]. The United States of America is one of the countries associated with the highest incidence of COVID-19 infection and death.

\section{Case presentation}

We present a case of a 32-year-old Hispanic man admitted to the hospital in December 2020 with ongoing fever, pleuritic chest pain, cough, and dyspnea for one week who was

Jasmeet Gill

Gilljasmeet30@gmail.com

Christopher Alan Hebert

Christopher.hebert@kahdallas.com

Gates Burton Colbert

Gates.colbert@bswhealth.org

1 Baylor University Medical Center at Dallas, 3500 Gaston Avenue, Barnett Tower, Ste 904, Dallas, TX 75246, USA

2 Division of Nephrology, Baylor University Medical Center at Dallas, Dallas, USA subsequently diagnosed with COVID-19 infection. His past medical history is significant for childhood leukemia treated at age six with anthracycline therapy resulting in an orthotopic heart transplant in 2015. In May 2020, he was diagnosed with antibody-mediated rejection with positive donor specific antibody for both Human Leukocyte Antigen (HLA) class I and II. He was treated with methylprednisone, plasma exchange, Bortezomib and IVIG. His last cardiology follow up showed stable cardiac allograft function, controlled hypertension, and chronic kidney disease stage 3 (baseline creatinine of $1.8-2.0 \mathrm{mg} / \mathrm{dL}$ ). Home medications included Mycophenolate $500 \mathrm{mg}$ BID, Prednisone $5 \mathrm{mg}$ daily, Sirolimus $3 \mathrm{mg}$ daily, Hydrochlorothiazide $12.5 \mathrm{mg}$ daily, Aspirin $81 \mathrm{mg}$ daily and Atorvastatin $40 \mathrm{mg}$ daily. On admission he had sinus tachycardia, blood pressure of $155 / 110 \mathrm{mmHg}$, was afebrile, and oxygen saturation on room air was $97 \%$.

Laboratory results demonstrated elevated inflammatory makers with markedly elevated ferritin: $2,581 \mathrm{ng} / \mathrm{mL}$ and CRP $3.6(0.0-0.3) \mathrm{mg} / \mathrm{dL}$. Other laboratory results (Table 1) included white blood cells: $4000 / \mu \mathrm{L}$, hemoglobin: $13.5 \mathrm{~g} /$ dL, platelets: 43,000/microL, BUN: $99 \mathrm{mg} / \mathrm{dL}$, creatinine: $7.75 \mathrm{mg} / \mathrm{dL}$, sodium: $135 \mathrm{meq} / \mathrm{L}$, potassium: $4.1 \mathrm{meq} / \mathrm{L}$, chloride: $104 \mathrm{meq} / \mathrm{L}$, bicarbonate: $20 \mathrm{meq} / \mathrm{L}$, anion gap: 11, and peripheral blood smear revealed $1+$ schistocytes. Chest computed tomography showed multifocal ground glass opacities affecting all lobes with consolidation consistent with COVID-19 pneumonia. He was started on broad spectrum antibiotics and intravenous dexamethasone $6 \mathrm{mg}$ every $12 \mathrm{~h}$. Urinalysis (Table 2) showed protein $2+$, blood $2+$, with RBC 2-3/HPF. Further labs revealed creatine kinase: 578 U/L, low 
Table 1 Chemistry Panel

\begin{tabular}{llc}
\hline Serum chemistry & & Reference range \\
\hline BUN & $\mathbf{9 9} \mathbf{~ m g / d L}$ & $6-24$ \\
Creatinine & $\mathbf{7 . 7 5} \mathbf{~ m g / d L}$ & $0.76-1.27$ \\
Sodium & $135 \mathrm{mEq} / \mathrm{L}$ & $134-144$ \\
Potassium & $4.1 \mathrm{mEq} / \mathrm{L}$ & $3.5-5.2$ \\
Chloride & $104 \mathrm{mEq} / \mathrm{L}$ & $97-108$ \\
Bicarbonate & $20 \mathrm{mEq} / \mathrm{L}$ & $18-29$ \\
Total protein & $8.6 \mathrm{~g} / \mathrm{dL}$ & $6.0-8.5$ \\
Albumin & $4.1 \mathrm{~g} / \mathrm{dL}$ & $3.5-5.5$ \\
Calcium & $9.6 \mathrm{mg} / \mathrm{dL}$ & $8.7-10.2$ \\
Phosphorus & $6.1 \mathrm{mg} / \mathrm{dL}$ & $2.5-4.5$ \\
Alkaline phosphatase & $85 \mathrm{mg} / \mathrm{dL}$ & $39-117$ \\
\hline
\end{tabular}

Bolded values are in abnormal range

Table 2 Urinalysis

\begin{tabular}{ll}
\hline Urinalysis & \\
\hline pH & $\mathbf{5 . 0}$ \\
Specific gravity & 1.016 \\
Glucose & Negative \\
Ketones & Negative \\
Protein & $2+$ \\
Nitrite & Negative \\
Occult blood & $\mathbf{3 +}$ \\
RBC urine & $\mathbf{3 / H P F}$ \\
Bacteria & none \\
\hline Bolded values are in abnormal \\
range
\end{tabular}

haptoglobin $<5$ (40-240 mg/dL), elevated LDH $1054 \mathrm{U} / \mathrm{L}$, normal fibrinogen, low fibrin degradation product $<5 \mu \mathrm{g} / \mathrm{ml}$, normal INR 0.8, and normal reticulocyte count. There was great concern for thrombotic microangiopathy due to the laboratory markers suggestive of intravascular hemolysis, worsening kidney failure, and thrombocytopenia. After multidisciplinary discussion with the cardiology, hematology, and nephrology teams, plasma exchange was initiated. He received daily plasma exchange (one plasma volume and no plasma substitutes were used) for 3 days without any improvement in serum creatinine and thrombocytopenia. Plasma exchange was discontinued on the 3rd hospital day as the ADAMTS13 activity test resulted in the normal range. Serum complements including $\mathrm{C} 3, \mathrm{C} 4$, and $\mathrm{CH} 50$ were normal, and ANCA and the antiphospholipid antibody panel were negative. He was also initiated on intermittent hemodialysis on the 3rd hospital day for azotemia. On the 7th hospital day platelet count decreased to $15,000 / \mu \mathrm{L}$ and schistocytes increased to $3+$ on blood smear. On the 10th hospital day he underwent a kidney biopsy which had 32 glomeruli, four of which were globally sclerotic. Eight of the glomeruli showed variable fibrin thrombi (Fig. 1) in some of the capillaries, both afferent and efferent, without endocapillary hypercellularity or inflammation. The capillary walls are single layered. There was no necrosis or crescent formation. There was some evidence of acute tubular injury but interstitial fibrosis/tubular atrophy was less than $10 \%$. Immunofluorescence was negative. The biopsy was consistent with mild thrombotic microangioapathy (TMA) with only mild chronic changes. He was continued on intermittent hemodialysis three times a week and initiated on Eculizumab $900 \mathrm{mg}$ weekly on hospital day 13. Laboratory values prior to, and after, Eculizumab can be seen below in Table 3. He was dialysis-free on hospital day 21. He was discharged on Eculizumab $900 \mathrm{mg}$ weekly for 2 more doses followed by $1200 \mathrm{mg}$ once and then $1200 \mathrm{mg}$ every two weeks. On discharge, platelets were normal $>150,000 / \mu \mathrm{L}$ and creatinine was $2.29 \mathrm{mg} /$ dL. Pre and post Eculizumab platelet count trend can be seen in Fig. 2. His respiratory status and oxygenation improved to pre-admission baseline without any oxygen requirements. On

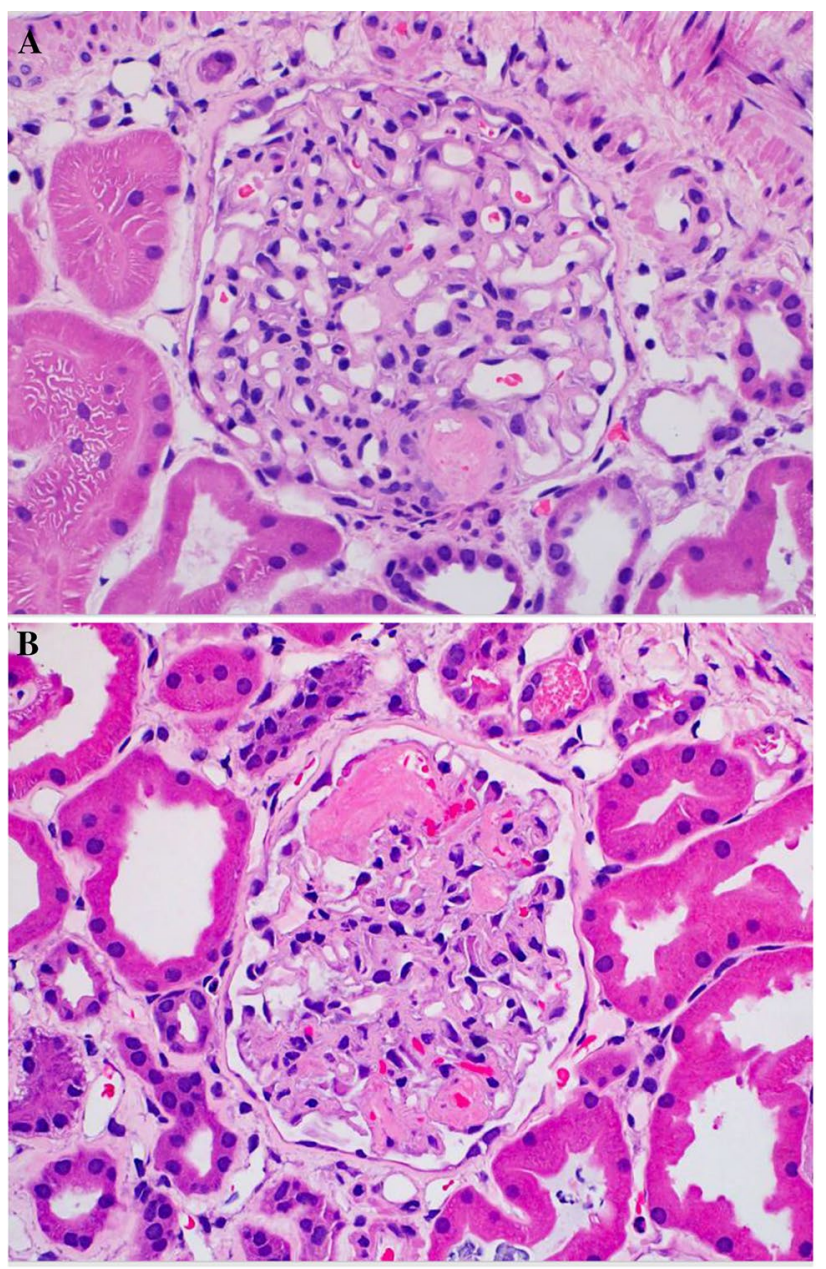

Fig. 1 Renal Biopsy Slides: Fibrin Thrombi in arteriole without endocapillary hypercellularity or inflammation consistent with thrombotic microangiopathy 
Table 3 Comparison of labs pre and post Eculizumab therapy

\begin{tabular}{lllll}
\hline Lab values & On admission & Pre-Eculizumab & $\begin{array}{l}\text { 7th day post Eculi- } \\
\text { zumab }\end{array}$ & Reference range \\
\hline BUN & $\mathbf{9 9}$ & $\mathbf{8 0}$ & $\mathbf{4 5}$ & $6-24 \mathrm{mg} / \mathrm{dL}$ \\
Creatinine & $\mathbf{7 . 7 5}$ & $\mathbf{7 . 1 3}$ & $\mathbf{4 . 8}$ & $0.76-1.27 \mathrm{mg} / \mathrm{dL}$ \\
Hemoglobin & 13.5 & 7.5 & 7.8 & $13.5-18.0 \mathrm{~g} / \mathrm{dL}$ \\
Platelet count & 56 & 28 & 105 & $140-400 \mathrm{k} / \mathrm{uL}$ \\
LDH & 1054 & 2076 & 583 & $85-245 \mathrm{U} / \mathrm{L}$ \\
Ferritin & 2581 & 827 & 472 & $26-388 \mathrm{ng} / \mathrm{ml}$ \\
\hline
\end{tabular}

Bolded values are in abnormal range

\section{Platelet count trend}

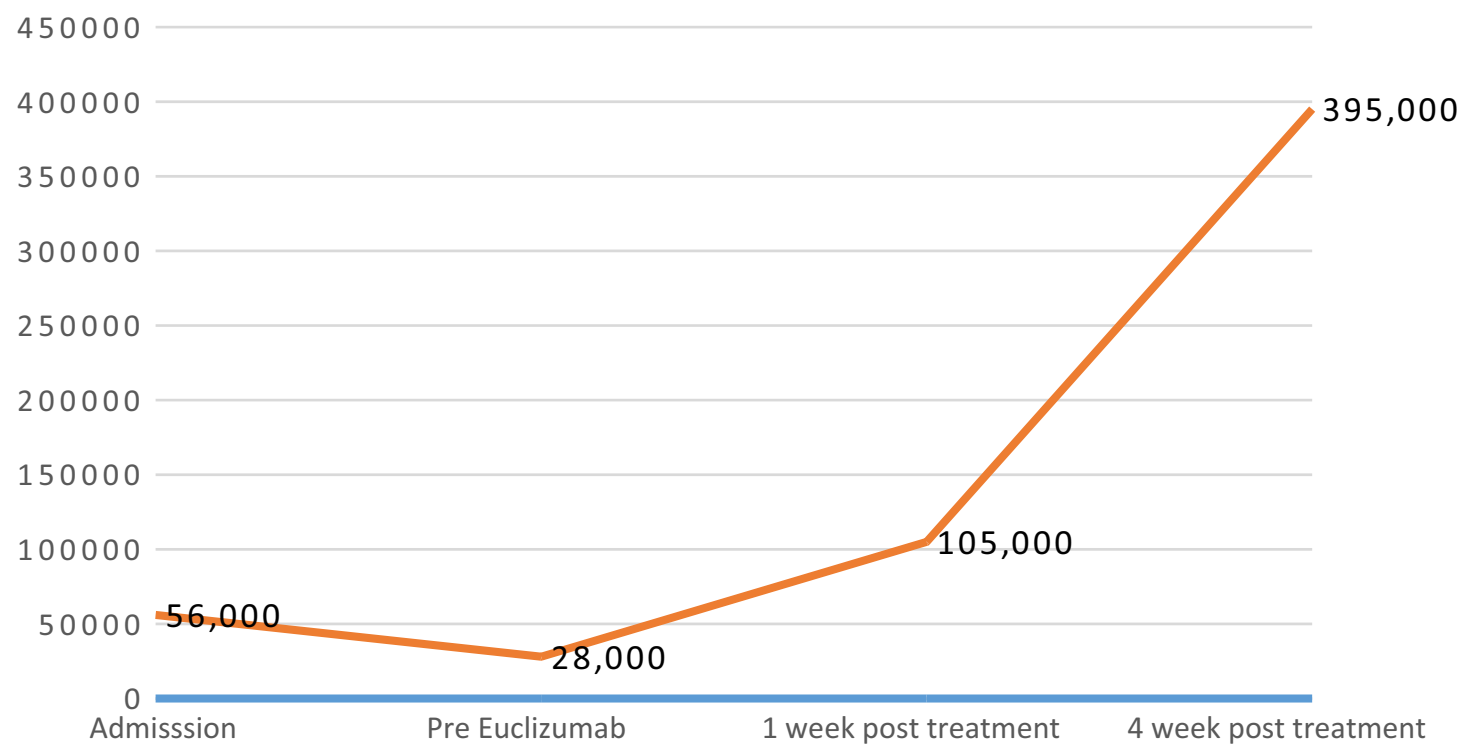

Fig. 2 Platelets count trend during hospitalization and pre and post Eculizumab

outpatient follow up after 6 months his creatinine improved to $1.42 \mathrm{mg} / \mathrm{dL}$. The patient continued to have proteinuria below $1 \mathrm{~g} /$ day but eGFR was holding stable. The patient transitioned to ravulizumab, for convenience of dosing, after 2 months and continued for at least 6 months as of publication of this manuscript.

Complement genetic testing panel was sent to the Mayo clinic laboratories and is negative for: ADAMTS13, CD46, CFB, CFH, CFHR1, CFHR3, CFHR5, CFI, C3, CFD, DGKE, PLG, THBD.

\section{Discussion}

The reported incidence of acute kidney injury in critically ill COVID-19 patients in New York has been as high as 36.6\% with the most common cause being ischemic acute tubular necrosis (ATN) [2]. The finding of acute tubular necrosis was confirmed on 26 kidney autopsy slides [4]. It has been reported that AKI in COVID-19 is a complex process including virus-mediated injury, cytokine storm, dysregulation of complements, hypercoagulation and microangiopathy [3].

Retrospective analysis of 6 autopsy slides by Diao et al. [5] from China showed severe ATN, C5b-9 deposition in the tubular lumen suggesting complement-mediated damage as normal kidney tissues do not have any C5-9 deposition. In 2003, during the SARS epidemic in China, blood and serum samples of 569 patients were studied and it was found that patients with low levels of serum mannose binding lectin (MBL) were more susceptible to SARS-CoV infection. MBL acts as an ante-antibody and inhibits antibody response [6]. In 2020, Gao et al. demonstrated increased serum C5a levels in severe COVID-19 infections and use of anti-C5a monoclonal antibody therapy (open label drug, phase 1 clinical trial). They reported two patients with severe respiratory illness who were found to have a decrease in 
oxygen requirement and a reduction in inflammatory markers post recombinant C5a antibody therapy [7]. The abovementioned studies suggested that complement system has a significant role in the COVID-19 disease process.

Another autopsy study of 5 patients with severe COVID19 infection lung and skin capillaries showed extensive deposits of the terminal component of C5b-9 complexes as well as C4d deposition [8]. The injury pattern was suggestive of activation of an alternative complement pathway leading to complement-mediated microvascular injury and thrombosis. Gralinski et al. studied mice infected with SARS-CoV and reported activation of $\mathrm{C} 3$ and its deposits in the lungs of these mice [9]. Diurno et al. from Italy reported four confirmed cases of SARS-CoV-2 with severe pneumonia or ARDS who were treated with Eculizumab in combination with hydroxychloroquine, antivirals and ceftriaxone. All four cases recovered with a marked drop in inflammatory markers within two weeks [10]. Based on the available data, Noris et al. [1] proposed the plausible hypothesis that cells infected and damaged by SARS-CoV-2 activate lectin and classical pathways and increase the production of terminal complement pathway C5a and C5b-9 which leads to endothelial dysfunction. The group has two ongoing compassionateuse protocols in the actively recruiting phase involving the administration of Eculizumab C5a inhibitor in COVID-19 patients (Clinicaltrials.gov: NCT04288713, NCT04346797).

Our search of English language literature on PubMed as of May 2021 showed less than 10 cases of thrombotic microangiopathy with COVID-19 infection including both pediatric and adult populations. In the majority of cases biopsy was deferred due to severity of illness and patients were treated based on clinical diagnosis. COVID-19 incidence is low in the pediatric population, however, among them there are at least two cases reported with complement-mediated TMA. The first patient, a 16-month-old boy, presented with COVID-19, new onset DKA, hemolytic anemia, thrombocytopenia and kidney failure. He was given a trial of fresh frozen plasma, however there was no improvement. His CH50 complement activity was low with elevated factor $\mathrm{H}$ and C5b-9 levels. He was started on Eculizumab and showed improvement in laboratory markers. Genetic testing was unremarkable [11]. Mahajan et al. reported a 14-year-old female with COVID-19 who was treated with Eculizumab for clinically suspected complement-mediated TMA. Serum C5b-9 was elevated. The patient was able to discontinue dialysis within $24 \mathrm{~h}$ of Eculizumab, and recovery of kidney function to baseline occurred in 3 weeks [12]. Ville et al. reported a case of atypical HUS relapse in a 28-year-old woman who was positive for COVID-19. Again, Eculizumab was started based on clinical and laboratory parameters and within 7 days of therapy hematological parameters and kidney function improved [13]. Another case of a 69-year-old female with severe COVID-19 illness had concern for TMA and underwent kidney biopsy which showed severe acute thrombotic microangiopathy. The patient was started on Eculizumab however she died after $48 \mathrm{~h}$ of initiating therapy due to worsening shock [14].

Based on the data presented above, it can be concluded that there is complement activation in COVID-19 illness which leads to systemic thrombotic microangiopathy in some patients. It has been reported in the past that TMA/ atypical HUS can relapse in the setting of viral illnesses like influenza. Some authors have suggested adding COVID-19 as a triggering factor for aHUS relapse. (It is a prime area of interest to explore use of Eculizumab in COVID-19 patients in prevention of severe illness and other serious manifestations in microvascular level.)

Sirolimus, the mTOR inhibitor, inhibits the production of VEGF which is required for endothelial cell survival. Thus, sirolimus can cause endotheliosis and trigger TMA (however risk in increased with combination of cyclosporine or tacrolimus). Most of the time TMAs caused by immunosuppressive medications are renal-limited [15]. Our patient had been on sirolimus for several months prior to this incident and in addition, since starting treatment he has continued sirolimus and has not had any ongoing symptoms. Hence, it is less likely that TMA was triggered by sirolimus alone as the presentation was systemic and not renal-limited. Our case is one of the few reported in the world of COVID-19 and biopsy-confirmed TMA with resolution of COVID-19 combined with Eculizumab. At the six month follow up visit our patient's renal function is stable with a creatinine level of $1.42 \mathrm{mg} / \mathrm{dL}$. He was switched to one infusion of Ravulizumab every eight weeks., Furthermore, this may be one of the first reported cases of COVID-19- induced HUS being treated with the newer complement inhibitor Ravulizumab. As of this manuscript writing, the patient is 6 months post initiation of treatment and ongoing aggressive treatment. (It is planned with coordination and education of the patient's wishes, to explore stopping complement inhibitor therapy between 6 and 12 months if the patient continues to have stable kidney function and no return of other symptoms.)

Author contribution JG: writing original draft, editing. CAH and GBC: review and editing. $\mathrm{CAH}$ and GBC: supervision and mentorship. Each author contributed important intellectual content during manuscript drafting or revision, accepts personal accountability for the author's own contributions, and agrees to ensure that questions pertaining to the accuracy or integrity of any portion of the work are appropriately investigated and resolved.

Funding There was no financial grant taken.

\section{Declarations}

Ethical approval The article does not contain any studies with human participants or animals performed by any of the authors. 
Conflict of interest No conflicts of interest, financial or other, exist.

Informed consent For this type of article formal informed consent is not required.

Consent for publication Yes.

\section{References}

1. Noris M, Benigni A, Remuzzi G (2020) The case of complement activation in COVID-19 multiorgan impact. Kidney Int 98:314-322

2. Hirsch JS, Ng JH, Ross DW, Sharma P, Shah HH, Barnett RL et al (2020) Acute kidney injury in patients hospitalized with COVID19. Kidney Int 98(1):209-218

3. Batlle D, Soler MJ, Sparks MA, Hiremath S, South AM, Welling PA, Swaminathan S (2020) Acute kidney injury in COVID-19: emerging evidence of a distinct pathophysiology. J Am Soc Nephrol 31(7):1380-1383

4. Su H, Yang M, Wan C, Yi LX, Tang F, Zhu HY et al (2020) Renal histopathological analysis of 26 postmortem findings of patients with COVID-19 in China. Kidney Int. 98(1):219-227

5. Diao B, Feng Z, Wang C, Wang H, Liu L, Wang C et al (2020) Human kidney is a target for novel severe acute respiratory syndrome coronavirus 2 (SARS-CoV-2) infection. MedRxiv. 9:221

6. Ip WE, Chan KH, Law HK, Tso GH, Kong EK, Wong WH et al (2005) Mannose-binding lectin in severe acute respiratory syndrome coronavirus infection. J Infect Dis 191(10):1697-1704

7. Gao T, Hu M, Zhang X, Li H, Zhu L, Liu H et al (2020) Highly pathogenic coronavirus $\mathrm{N}$ protein aggravates lung injury by MASP-2-mediated complement over-activation. MedRxiv

8. Magro C, Mulvey JJ, Berlin D et al (2020) Complement associated microvascular injury and thrombosis in the pathogenesis of severe COVID-19 infection: a report of five cases. Transl Res 220:1-13. https://doi.org/10.1016/j.trsl.2020.04.007
9. Gralinski LE, Sheahan TP, Morrison TE, Menachery VD, Jensen K, Leist SR, Whitmore A, Heise MT, Baric RS (2018) Complement activation contributes to severe acute respiratory syndrome coronavirus pathogenesis. MBio 9(5):e01753-18. https://doi.org/ 10.1128/mBio.01753-18

10. Diurno F, Numis FG, Porta G, Cirillo F, Maddaluno S, Ragozzino A, De Negri P, Di Gennaro C, Pagano A, Allegorico E, Bressy L, Bosso G, Ferrara A, Serra C, Montisci A, D’Amico M, Schiano Lo Morello S, Di Costanzo G, Tucci AG, Marchetti P et al (2020) Eculizumab treatment in patients with COVID-19: preliminary results from real life ASL Napoli 2 Nord experience. Eur Rev Med Pharmacol Sci 24(7):4040-4047

11. Alizadeh F, O'Halloran A, Alghamdi A, Chen C, Trissal M, Traum A, DeCourcey D (2021) Toddler with new onset diabetes and atypical hemolytic-uremic syndrome in the setting of COVID19. Pediatrics 147(2):e2020016774

12. Mahajan R, Lipton M, Broglie L, Jain NG, Uy NS (2020) Eculizumab treatment for renal failure in a pediatric patient with COVID-19. J Nephrol 33(6):1373-1376

13. Safak S, Aksoy E, Dirim AB, Demir E, Garayeva N, Oto OA et al (2021) Successful treatment of a COVID-19 patient with thrombotic microangiopathy. Clin Kidney J 14(4):1287-1288

14. Jhaveri KD, Meir LR, Chang BSF, Parikh R, Wanchoo R, BarillaLaBarca ML et al (2020) Thrombotic microangiopathy in a patient with COVID-19. Kidney Int 98(2):509-512

15. Sartelet H, Toupance O, Lorenzato M, Fadel F, Noel LH, Lagonotte E, Birembaut P, Chanard J, Rieu P (2005) Sirolimusinduced thrombotic microangiopathy is associated with decreased expression of vascular endothelial growth factor in kidneys. Am J Transplant 5(10):2441-2447. https://doi.org/10.1111/j.1600-6143. 2005.01047.x

Publisher's Note Springer Nature remains neutral with regard to jurisdictional claims in published maps and institutional affiliations. 